\title{
Alterations in mitosis and cell cycle progression caused by a mutant lamin A known to accelerate human aging
}

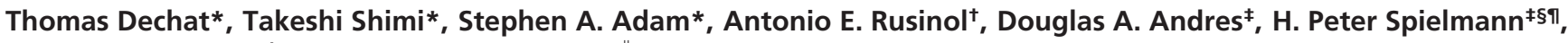 \\ Michael S. Sinensky ${ }^{\dagger}$, and Robert D. Goldman*\|

\begin{abstract}
*Department of Cell and Molecular Biology, Feinberg School of Medicine, Northwestern University, 303 East Chicago Avenue, Chicago, IL 60611; ${ }^{\dagger}$ Department of Biochemistry and Molecular Biology, James H. Quillen College of Medicine, East Tennessee State University, Box 70581, Johnson City, TN 37614; and Departments of ₹ Molecular and Cellular Biochemistry and ${ }^{\S}$ Chemistry and ${ }^{9}$ Kentucky Center for
\end{abstract} \\ Structural Biology, University of Kentucky, 741 South Limestone, Lexington, KY 40536
}

Communicated by Francis S. Collins, National Institutes of Health, Bethesda, MD, February 2, 2007 (received for review December 19, 2006)

Mutations in the gene encoding nuclear lamin A (LA) cause the premature aging disease Hutchinson-Gilford Progeria Syndrome. The most common of these mutations results in the expression of a mutant LA, with a $\mathbf{5 0}$-aa deletion within its $\mathrm{C}$ terminus. In this study, we demonstrate that this deletion leads to a stable farnesylation and carboxymethylation of the mutant LA (LA $\Delta 50 /$ progerin). These modifications cause an abnormal association of LA $\Delta 50$ / progerin with membranes during mitosis, which delays the onset and progression of cytokinesis. Furthermore, we demonstrate that the targeting of nuclear envelope/lamina components into daughter cell nuclei in early $G_{1}$ is impaired in cells expressing LA $\Delta \mathbf{5 0}$ / progerin. The mutant LA also appears to be responsible for defects in the retinoblastoma protein-mediated transition into S-phase, most likely by inhibiting the hyperphosphorylation of retinoblastoma protein by cyclin D1/cdk4. These results provide insights into the mechanisms responsible for premature aging and also shed light on the role of lamins in the normal process of human aging.

cell division | nuclear lamins | nuclear structure | progeria

protein farnesylation

$\mathbf{H}$ utchinson-Gilford progeria syndrome (HGPS) is an early onset aging disease $(1,2)$ most commonly caused by a heterozygous mutation in the lamin A (LA) gene ( $L M N A, 1824$ $\mathrm{C} \rightarrow \mathrm{T})(3,4)$. This mutation introduces a splice site, resulting in the expression of a mutant LA (LA $\Delta 50$ /progerin) $(5,6)$ that is missing 50 aa near its $C$ terminus. HGPS patients carrying this mutation experience accelerated aging symptoms, including loss of s.c. fat, growth retardation, hair loss, skeletal hypoplasia and dysplasia, osteoporosis, and arteriosclerosis. Patients usually die at an average of 15 years of age from heart attacks or strokes (7). Other than HGPS, various diseases, including muscular dystrophies and lipodystrophies, have been linked to mutations in LMNA (8).

Lamins are intermediate filament proteins located in the nuclear lamina and throughout the nucleoplasm $(9,10)$. In humans, lamins are divided into $\mathrm{A}$ and $\mathrm{B}$ types. The major A-type lamins, LA and lamin C (LC), are derived from a single gene by alternative splicing, whereas B-type lamins (LB) are encoded by different genes. Lamins $\mathrm{A}$ and $\mathrm{B}$ are modified at their C-terminal -CAAX box in a series of steps involving farnesylation of the cysteine residue, cleavage of - AAX, and carboxymethylation $(11,12)$. Whereas LB is permanently farnesylated/carboxymethylated, LA is cleaved by Zmpste24, a zinc-metalloproteinase, removing another 15 aa from the $\mathrm{C}$ terminus, including the farnesylated/carboxymethylated cysteine (13-15). Because the cleavage site for Zmpste24 is missing in LA $\Delta 50$ /progerin, it is thought, but has yet to be demonstrated biochemically, that it is stably farnesylated (16).

In interphase cells, the expression of LA $\Delta 50$ /progerin leads to nuclear lobulation, thickening of the lamina, genome instability,
DNA repair defects, changes in histone methylation, and loss of heterochromatin $(5,17-19)$. However, the impact of LA $\Delta 50$ / progerin on mitosis and its consequences for daughter cells entering $G_{1}$ have not been determined. An initial insight into changes in early $G_{1}$ came from studies of HeLa cells expressing GFP-LA $\Delta 50$ /progerin, in which this mutant protein is abnormally retained in cytoplasmic structures after nuclear assembly is completed (5). Here, we provide further insights into the composition of these structures and the impact of LA $\Delta 50$ / progerin on cell division, nuclear assembly, and the cell cycle.

\section{Results}

First, we verified that cytoplasmic structures similar to those seen in GFP-LA $\Delta 50$ /progerin transfected HeLa cells (5) were present in HGPS patient fibroblasts. We found that daughter cells in early $\mathrm{G}_{1}$ contained abnormal cytoplasmic structures enriched in LA/C (Fig. $1 A a, A d$, and $A g$ ). These structures also react with anti-LA (Fig. $1 A a-A c$ ), which does not crossreact with $\mathrm{LA} \Delta 50$ /progerin [supporting information (SI) Fig. 6], and with anti-LB and anti-emerin, an integral nuclear membrane protein known to bind to LA (20) (Fig. $1 A d-A i)$. In control fibroblasts, $\mathrm{LA} / \mathrm{C}, \mathrm{LB}$, and emerin are sequestered in the nucleus in early $G_{1}$ as expected (data not shown). These observations suggest that LA $\Delta 50$ /progerin causes the aberrant retention of nuclear membrane and lamina components in the cytoplasm well into $G_{1}$. At times after cell division (e.g., late $G_{1}, S$ ), all detectable LA $\Delta 50 /$ progerin, $\mathrm{LA} / \mathrm{C}, \mathrm{LB}$, and emerin are located in the nucleus (data not shown and ref. 5).

The retention of nuclear components in the cytoplasm in early $\mathrm{G}_{1}$ in HGPS fibroblasts suggests that progression through the cell cycle is altered, which could affect the $\mathrm{G}_{1} / \mathrm{S}$ transition (5). To test this possibility, we examined the retinoblastoma protein $(\mathrm{Rb})$ in HGPS cell nuclei, because it binds to LA (21) and its hyperphosphorylated form (phosphoRb) is required for the $\mathrm{G}_{1} / \mathrm{S}$ transition (22). In the majority $(94.2 \%, n=53)$ of HGPS cells with highly lobulated nuclei and a thickened lamina (Fig. $1 B a-B c$, red) (5), we could not detect phosphoRb by using an antibody against the phosphorylated $\mathrm{Rb}$

Author contributions: T.D., T.S., A.E.R., M.S.S., and R.D.G. designed research; T.D., T.S., A.E.R., and M.S.S. performed research; S.A.A., D.A.A., and H.P.S. contributed new reagents analytic tools; T.D., T.S., A.E.R., M.S.S., and R.D.G. analyzed data; and T.D., A.E.R., M.S.S., and R.D.G. wrote the paper.

The authors declare no conflict of interest.

Abbreviations: AG, anilinogeraniol; FTase, farnesyl transferase; FTI, FTase inhibitor; HGPS, Hutchinson-Gilford progeria syndrome; LA, lamin A; LB, B-type lamins; LC, lamin C; LA $\Delta 50$ / progerin, mutant LA in HGPS cells; NEBD, nuclear envelope breakdown; Rb, retinoblastoma protein.

ITo whom correspondence should be addressed. E-mail: r-goldman@northwestern.edu.

This article contains supporting information online at www.pnas.org/cgi/content/full/ 0700854104/DC1.

() 2007 by The National Academy of Sciences of the USA 
A

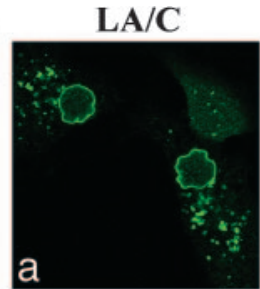

LA/C

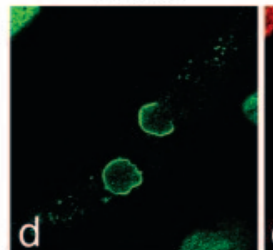

LA/C

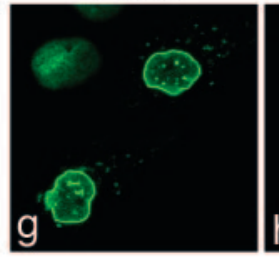

LA

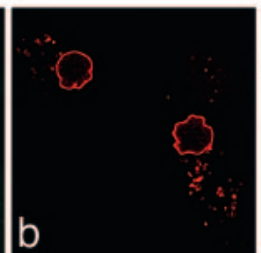

LB

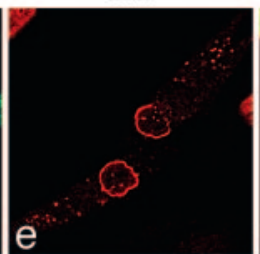

Emerin

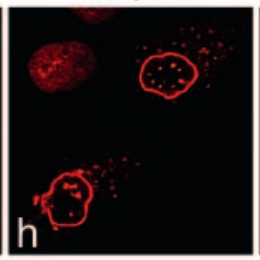

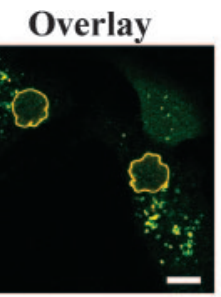

Overlay

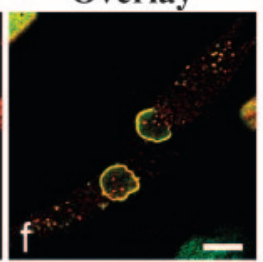

Overlay

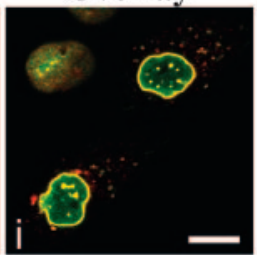

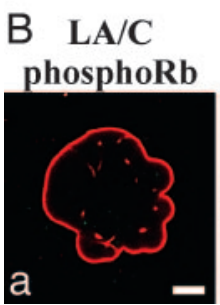

LA/C

Rb

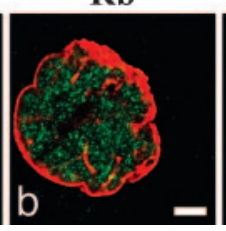

LA/C cyclin D1

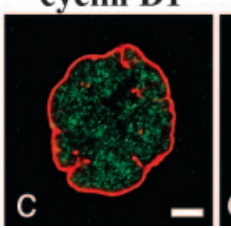

LA/C phosphoRb

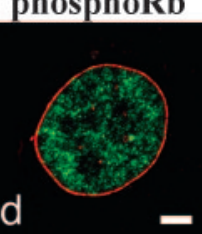

Fig. 1. Cells from HGPS patients expressing LA $\Delta 50 /$ progerin contain abnormal cytoplasmic structures enriched in nuclear lamina/envelope components in early $\mathrm{G}_{1}$. (A) Mid- to late-passage (p) (5) HGPS fibroblasts (HGADFN003, p18) were fixed in $3 \%$ paraformaldehyde and processed for immunofluorescence with anti-LA/C ( $a, d$, and $g)$, anti-LA (b), anti-LB (e), and anti-emerin (h). Early $\mathrm{G}_{1}$ cells displayed abnormal cytoplasmic structures containing $\mathrm{LA} / \mathrm{C}(a, c, d, f$, $g$, and $i)$, LA ( $b$ and $c), \mathrm{LB}$ (e and $f$ ), and emerin ( $h$ and $i)$. These structures are also seen in HGPS fibroblasts from another patient (HGADFN127, p16) but not in control fibroblasts [AG08470 (p17) and AG09309 (p18)] (data not shown). Confocal images are shown. (Scale bars, $10 \mu \mathrm{m}$.) (B) Late passage HGPS fibroblasts (HGADFN003, p22) were methanol fixed and processed for double immunofluorescence by using anti-LA/C ( $a-d$, red) and either anti-phosphoRb(Ser-807/811) ( $a$ and $d$, green), anti-Rb ( $b$, green), or anti-cyclin D1 ( $c$, green). Confocal images are shown. (Scale bars, $5 \mu \mathrm{m}$.)

residues Ser-807/811 (Fig. 1Ba, green). Patients' cells with normally shaped nuclei and a typical lamina frequently $(38.5 \%, n=53)$ contained phosphoRb (Fig. $1 B d$, green), indicating that they are in either $\mathrm{S}$ or $\mathrm{G}_{2} / \mathrm{M}$ (22). However, $\mathrm{Rb}$ was present in the highly lobulated nuclei (Fig. $1 B b$, green), suggesting that the absence of phosphoRb is not due to degradation. Cyclin D1, a cofactor for cdk 4 that phosphorylates $\mathrm{Rb}$ at Ser-807/811 (23), is present in the lobulated nuclei (Fig. $1 B c$, green). This suggests that LA $\Delta 50$ / progerin impairs the Rb-mediated $\mathrm{G}_{1} / \mathrm{S}$ transition because of inhibition of cdk 4 activity.

To gain insights into the mechanisms responsible for the formation of the abnormal cytoplasmic nuclear membrane/ lamina components, we determined whether LA $\Delta 50 /$ progerin is farnesylated. This posttranslational modification might cause an abnormal affinity for membranes $(11,24)$. Previously, massspectrometry was used to detect CAAX box modifications on gel purified pre-LA (14). Because we have encountered technical
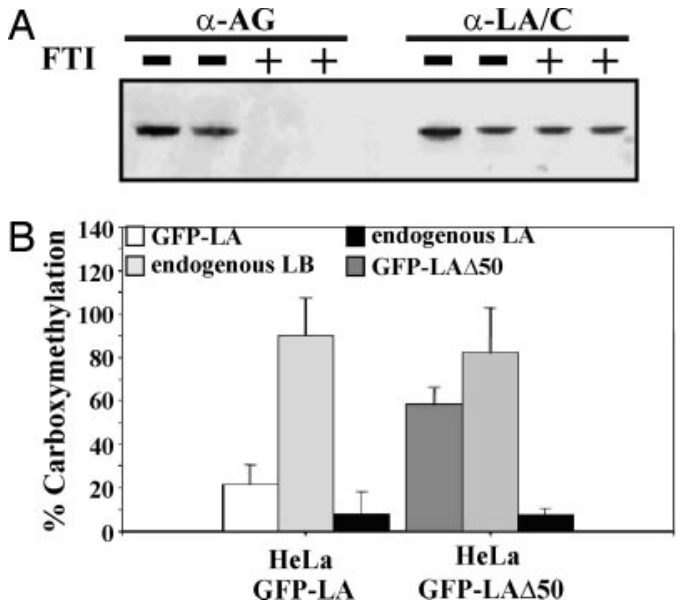

Fig. 2. LA $\Delta 50 /$ progerin is farnesylated and carboxymethylated. (A) HeLa cells expressing GFP-LA $\Delta 50 /$ progerin were incubated with the farnesol analogue, AG, and with (+FTI) or without ( $-\mathrm{FTI}) 3 \mu \mathrm{M} \mathrm{FTI-277.} \mathrm{GFP-LA} \Delta 50 /$ progerin was immunoprecipitated with anti-GFP and analyzed by immunoblotting, using either anti-AG $(\alpha-A G)$ or anti-LA/C $(\alpha$-LA/C). The results of two experiments $( \pm \mathrm{FTI})$ are shown. $(B)$ HeLa cells expressing GFP-LA $\Delta 50 /$ progerin or GFP-LA were incubated with $\left(\left[\right.\right.$ methyl $\left.\left.{ }^{3} \mathrm{H}\right]\right)$ methionine for $16-20 \mathrm{~h}$. After incubation, proteins were immunoprecipitated consecutively with antibodies against LB, LA, and EGFP, separated on SDS/PAGE, and identified by immunoblotting. Protein bands $(n=3)$ were excised, and carboxymethylation was determined as described in Materials and Methods.

difficulties applying this approach to LA $\Delta 50$ /progerin, we have used an alternative assay developed to detect the farnesylation of a CAAX protein (25). In this assay, a farnesol analogue, 8-anilinogeraniol (AG), is used as a prosubstrate for the farnesyl transferase (FTase). Detection of the unnatural lipid is achieved by immunoblotting with anti-AG. An important control to verify that an FTase is mediating incorporation of AG is inhibition with the FTase inhibitor (FTI), FTI-277. By using this approach, GFP-LA $\Delta 50 /$ progerin was immunoprecipitated from HeLa cells incubated with 8-AG and analyzed by immunoblotting (Fig. 2A). In the absence of the FTI (FTI-), a band of the expected molecular weight was detected with anti-AG $(\alpha-\mathrm{AG})$ and antiLA/C $(\alpha$-LA/C). However, when cells were treated with FTI-277 before immunoprecipitation (FTI+), only $\alpha$-LA/C showed immunoreactivity. These results demonstrate that LA $\Delta 50$ /progerin is farnesylated.

Verification of carboxymethylation of LA $\Delta 50 /$ progerin was obtained by means of a base-release assay (12), which also provides a relative measure of the amount of LA $\Delta 50$ /progerin that is completely processed at the -CAAX box. For this purpose, HeLa cells expressing GFP-LA $\Delta 50$ /progerin or GFP-LA were incubated with $\left(\left[\right.\right.$ methyl $\left.\left.-{ }^{3} \mathrm{H}\right]\right)$ methionine and used for immunoprecipitation of endogenous LA, LB, GFP-LA, and GFP-LA $\Delta 50 /$ progerin. The immunoprecipitated proteins were further analyzed (see Materials and Methods). The results of these experiments show that GFP-LA $\Delta 50$ /progerin is carboxymethylated to about the same extent as LB, whereas endogenous LA and GFP-LA are not carboxymethylated (Fig. 2B).

To determine the effects of the permanent state of farnesylation of LA $\Delta 50 /$ progerin, GFP-LA $\Delta 50 /$ progerin expression was studied in HeLa cells. During interphase, GFP-LA $\Delta 50$ /progerin could not be detected in the nucleoplasm (SI Fig. 7). Rather, it localized exclusively to the nuclear lamina region, most likely anchored to the inner nuclear membrane because of farnesylation $(11,16,24)$. In contrast, GFP-LA was also present as a nucleoplasmic veil in interphase nuclei (SI Fig. 7). We also determined the impact of the membrane association of LA $\Delta 50$ / progerin during mitosis. In normal cells, lamin depolymerization 

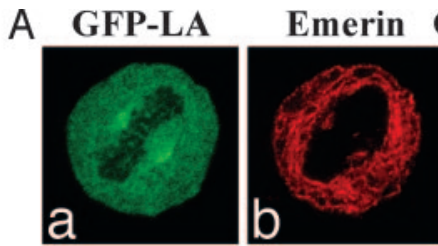

GFP-LA $\Delta 50$
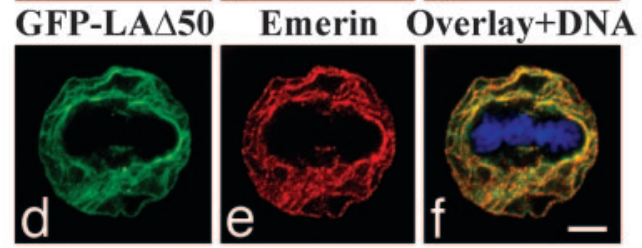
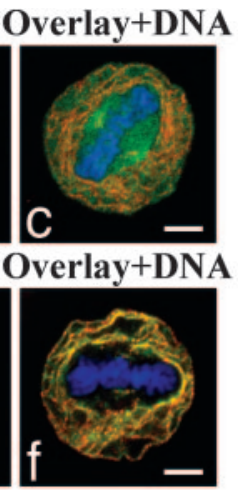

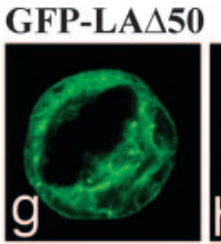

GFP-LA $\Delta 50$

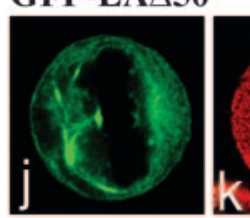

LB Overlay+DNA

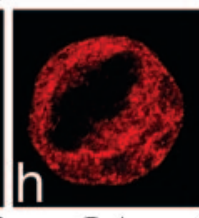

LA

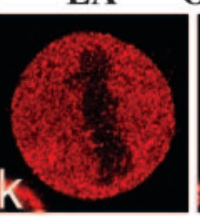

B

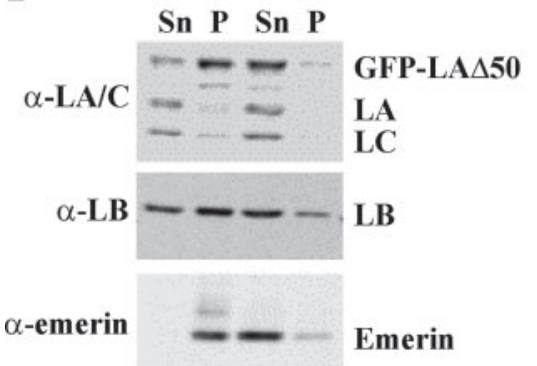

C

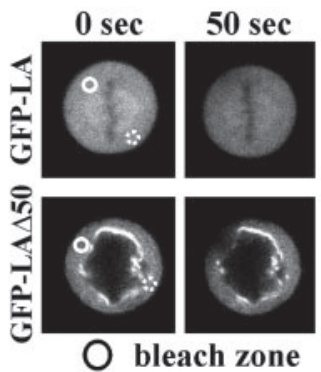

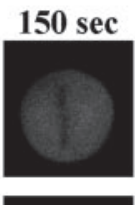

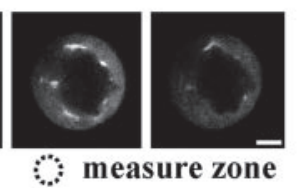

D

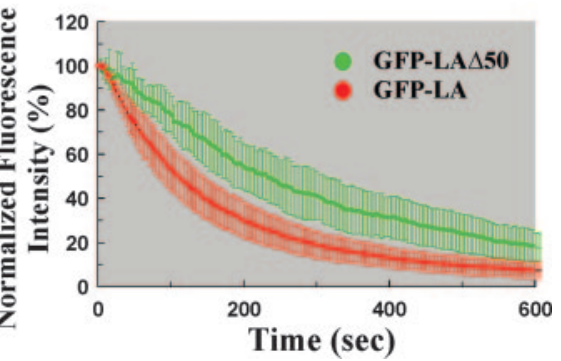

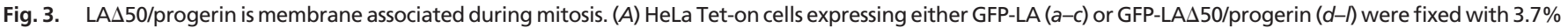

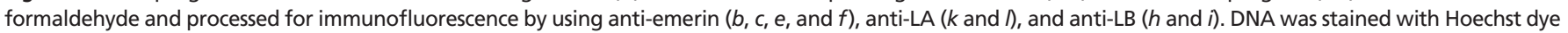

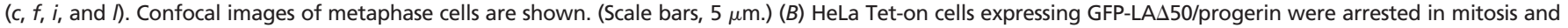

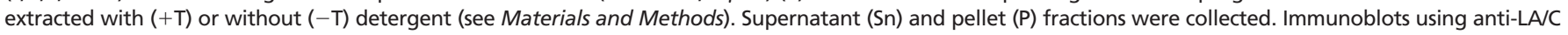

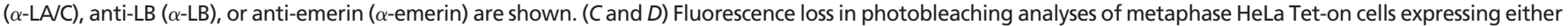

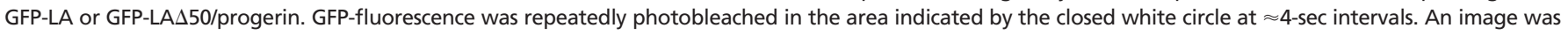

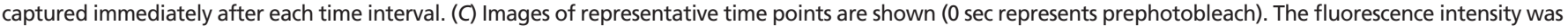

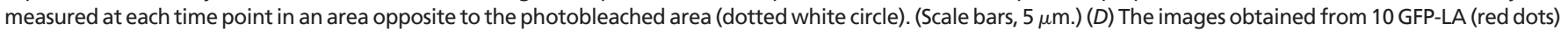

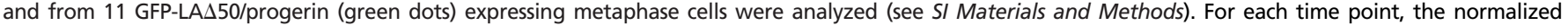
fluorescence intensity measurements were averaged and plotted as a function of time. Error bars are indicated for each time point.

takes place during nuclear envelope breakdown (NEBD) (10, 26). Whereas depolymerized LA/C is dispersed throughout the cytoplasm in metaphase, LB remains mainly membrane associated (26). Studies of mitotic HeLa cells expressing either GFP-LA or GFP-LA $\Delta 50 /$ progerin revealed that GFP-LA $\Delta 50$ / progerin colocalized with emerin and LB in membranous structures in metaphase (Fig. $3 A d-A i$ ), but not with endogenous LA (Fig. $3 A j-A l$ ). Similar structures are also detected in metaphase HGPS cells with a specific LA $\Delta 50$ /progerin antibody [see accompanying paper by Cao et al. (27)]. As expected, GFP-LA was uniformly distributed throughout the cytoplasm, sparing only the chromosomes, and it did not colocalize with emerin or LB (Fig. $3 A a-A c$ and data not shown). In addition, GFP-LA appeared to be more concentrated in the metaphase spindle in close proximity to microtubules (SI Fig. 8), whereas GFP-LA $\Delta 50$ /progerin appeared to be greatly reduced in this region (SI Fig. 8). Upon the addition of FTI-277, the association of GFP-LA $\Delta 50$ /progerin with membranes was no longer detectable in mitotic cells (SI Fig. 8). Rather, it appeared to be uniformly distributed and indistinguishable from GFP-LA in metaphase cells. Under these conditions, LA $\Delta 50 /$ progerin also appeared to be more concentrated in metaphase spindles as was the case for GFP-LA (SI Fig. 8 ). These data are especially interesting, because it has recently been shown that lamins are required for the formation of the mitotic spindle matrix (28).

Other evidence supporting the association of LA $\Delta 50 /$ progerin with membranes during mitosis was derived from biochemical fractionation studies. Mitotically arrested HeLa cells expressing GFP-LA $\Delta 50 /$ progerin were lysed, and supernatant (Sn) and pellet $(\mathrm{P})$ fractions were collected in the presence or absence of Triton X-100 (see Materials and Methods). Analysis of these fractions by immunoblotting revealed that, in the absence of detergent, the vast majority of GFP-LA $\Delta 50$ /progerin, LB, and emerin were in the insoluble pellet (Fig. $3 B,-\mathrm{T}$ ). In contrast, most $\mathrm{LA} / \mathrm{C}$ was in the soluble supernatant (Fig. $3 B,-\mathrm{T}$ ). Upon the addition of Triton X-100, there was a shift of GFP-LA $\Delta 50$ / progerin, $\mathrm{LB}$ and emerin to the soluble fraction (Fig. $3 B,+\mathrm{T}$ ). In control cells expressing GFP-LA, almost all GFP-LA and $\mathrm{LA} / \mathrm{C}$ remained in the soluble fraction under both conditions (data not shown). These results further demonstrate that LA $\Delta 50$ / progerin is abnormally attached to membranes during mitosis.

We also determined whether the process of GFP-LA $\Delta 50$ / progerin disassembly during NEBD differed from that of GFPLA, using live imaging of HeLa cells. In control cells, GFP-LA was located in the nuclear periphery and the nucleoplasm during the early stages of NEBD. As NEBD progressed, GFP-LA became uniformly distributed throughout the cytoplasm (SI Movie 1). In contrast, GFP-LA $\Delta 50$ /progerin was not detected within the nucleoplasm in cells at any time during NEBD. Rather, it was redistributed from the nuclear periphery to cytoplasmic membrane structures (SI Movie 2).

We next compared the dynamic properties of GFP-LA $\Delta 50$ / progerin with GFP-LA during metaphase in HeLa cells, using fluorescence loss in photobleaching assays. These assays demonstrated that the GFP-LA signal decreased uniformly throughout the entire cell (Fig. 3C Upper), whereas the GFP-LA $\Delta 50$ / progerin signal was lost in a more gradual fashion away from the bleach zone (Fig. 3C Lower). The time for loss of $\approx 50 \%$ 

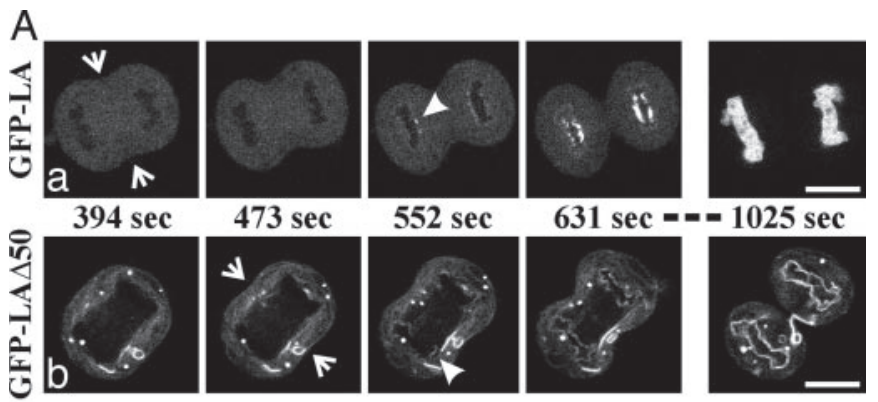

$631 \mathrm{sec}=-1025 \mathrm{sec}$
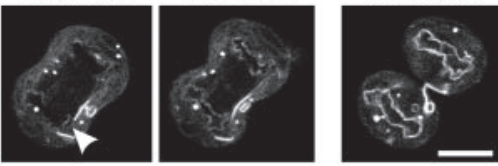

B

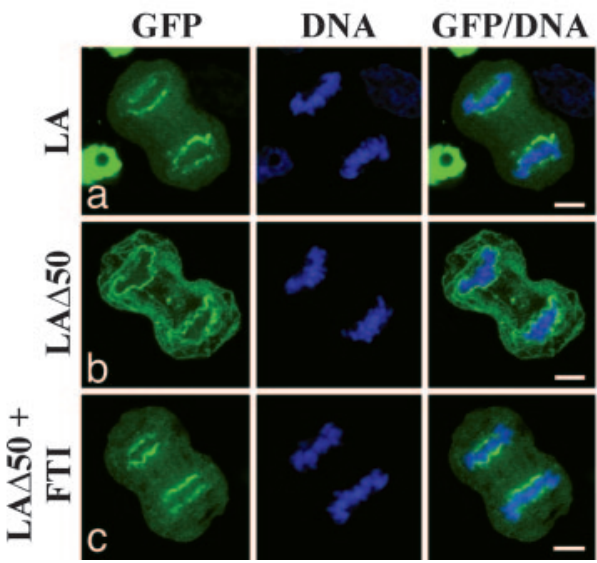

Fig. 4. LA $\Delta 50 /$ progerin re-localizes exclusively to the nuclear periphery at the end of mitosis and delays cytokinesis and nuclear envelope reassembly. (A) HeLa Tet-on cells expressing either GFP-LA (a) or GFP-LA $\Delta 50 /$ progerin (b) were followed by time-lapse microscopy from the metaphase/anaphase transition ( $0 \mathrm{sec})$ into $\mathrm{G}_{1}$. Confocal images were acquired at $\approx 79$-sec intervals (see SI Materials and Methods). Representative time points for the onset of cytokinesis (arrows) and for the initial localization of the GFP-fusion proteins to the newly forming sister nuclei (arrowheads) are shown (394-631 sec), as well as the time when most of the GFP-LA was located in the nucleus $(1,025 \mathrm{sec})$. (Scale bars, $10 \mu \mathrm{m}$.) (B) GFP-LA(a) and GFP-LA $\Delta 50 /$ progerin- $(b)$ expressing HeLa Tet-on cells were fixed with $3.7 \%$ formaldehyde, and DNA was stained with Hoechst dye. In addition, GFP$\mathrm{LA} \Delta 50$ /progerin expressing cells were incubated with FTI-277 before fixation (c). Confocal images of telophase cells are shown. (Scale bars, $5 \mu \mathrm{m}$.)

GFP-LA $\Delta 50 /$ progerin fluorescence was $\approx 232 \mathrm{sec}$, compared with $\approx 111 \mathrm{sec}$ for GFP-LA (Fig. $3 D$ ). These results suggest that the mobility of GFP-LA $\Delta 50$ /progerin is constrained because of its association with membranes, whereas GFP-LA can freely diffuse throughout the cytoplasm.

To determine whether the attachment of LA $\Delta 50 /$ progerin to membranes during mitosis leads to mitotic defects that could contribute to the phenotype seen in HGPS fibroblasts in early $\mathrm{G}_{1}$, time-lapse studies beginning with the metaphase/anaphase transition until late cytokinesis were carried out on live HeLa cells expressing GFP-LA $(n=9)$ or GFP-LA $\Delta 50 /$ progerin $(n=$ 8). First, we determined the time taken from the metaphase/ anaphase transition to the initiation of the cleavage furrow, which represents the onset of cytokinesis (see arrows in Fig. 4A). The average time in GFP-LA-expressing cells was $412 \pm 35 \mathrm{sec}$, and in cells expressing GFP-LA $\Delta 50$ /progerin it was $483 \pm 51 \mathrm{sec}$, to the initiation of cleavage (Fig. $4 A$ and SI Movies 3 and 4). This finding reflects a delay of $\approx 17 \%(P=0.0039)$ in the time of onset of cytokinesis in cells expressing GFP-LA $\Delta 50$ /progerin. Next, we determined the timing of the relocalization of both the mutant and wild type proteins to segregating sister chromatids. The two proteins did not show significant differences in the timing of their initial relocalization $[543 \pm 26 \mathrm{sec}$ for GFP-LA vs. $522 \pm 41 \mathrm{sec}$ for GFP-LA $\Delta 50 /$ progerin $(P=0.2205)$; see arrowheads in Fig. $4 A]$. However, GFP-LA $\Delta 50 /$ progerin relocated to and remained
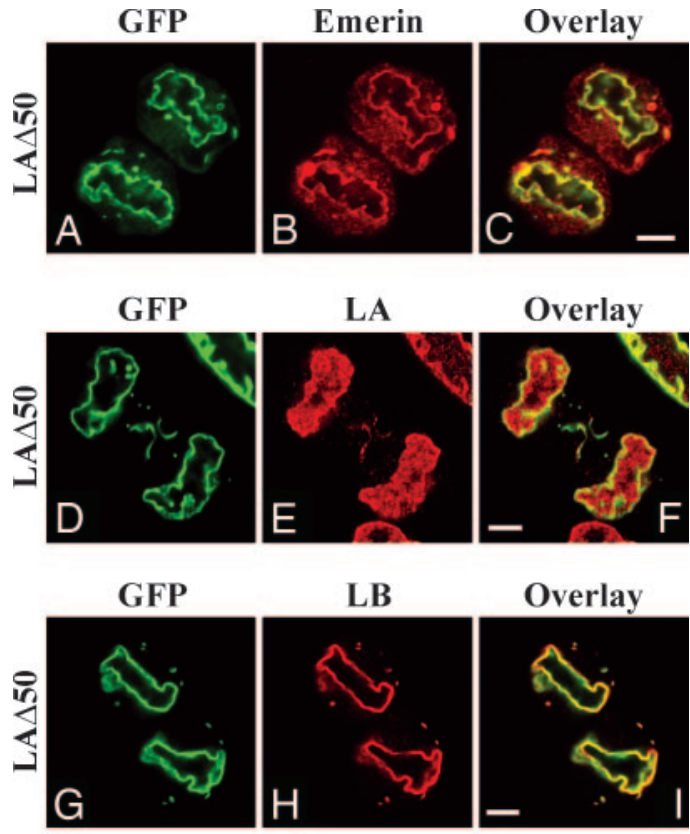

Overlay
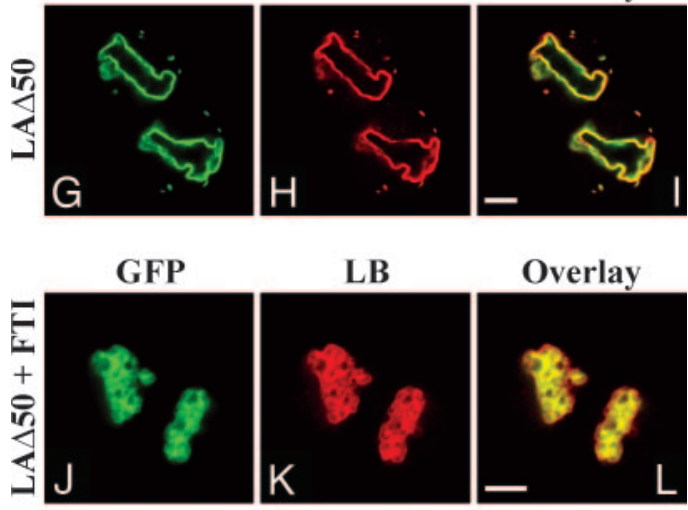

Overlay
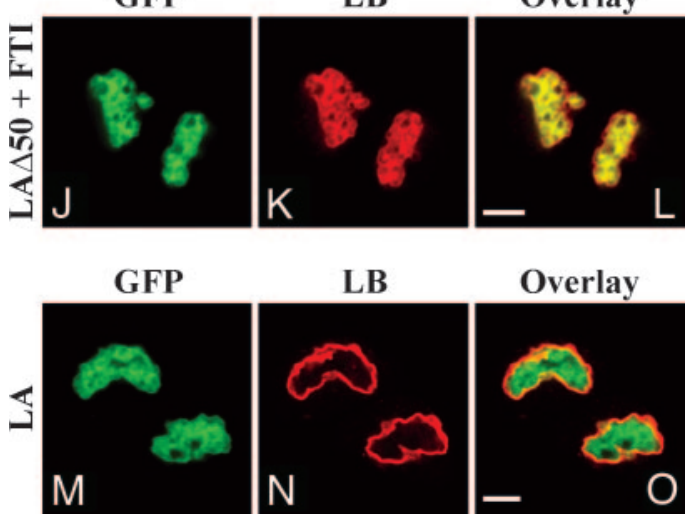

Fig. 5. $L A$ and $L B$ are retained in the cytoplasm in early $G_{1}$ cells expressing GFP-LA $\Delta 50$ /progerin. HeLa Tet-on cells expressing either GFP-LA $\Delta 50 /$ progerin $(A-I)$ or GFP-LA $(M-O)$ were fixed with $3.7 \%$ formaldehyde and processed for immunofluorescence by using anti-emerin ( $B$ and $C)$, anti-LA ( $E$ and $F$ ) and anti-LB $(H, I, K, L, N$, and $O)$. In addition, GFP-LA $\Delta 50 /$ progerin expressing cells were incubated with FTI-277 before fixation $(J-L)$. Confocal images of late telophase/early $\mathrm{G}_{1}$ cells are shown. (Scale bars, $5 \mu \mathrm{m}$.)

exclusively at the periphery of segregating sister chromatids (Fig. $4 A b$ and $B b$ and SI Movie 4), a pattern similar to that seen for LB (29); whereas GFP-LA relocalized initially to the "core regions" of sister chromatids (29) and subsequently accumulated throughout the forming nucleoplasm (Figs. $4 A a$ and $B a$ and $5 m$, and SI Movie 3). The addition of FTI-277 to GFP-LA $\Delta 50$ / progerin-expressing cells caused a reversion to patterns seen in GFP-LA-expressing cells (Figs. $4 B c$ and $5 J$ ).

Regarding the timing of the completion of nuclear relocalization, GFP-LA $\Delta 50 /$ progerin was retained in the cytoplasm in late telophase/early $\mathrm{G}_{1}$ compared with GFP-LA, which was similar to the observations made in HGPS cells. In cells expressing GFPLA, it took $1,060 \pm 42 \mathrm{sec}$ from the metaphase/anaphase transition until all detectable GFP-LA was located in the newly formed nuclei (Fig. $4 A a, 1,025 \mathrm{sec}$ ). However, at this same time point there was still a significant amount of GFP-LA $\Delta 50$ / progerin present in the cytoplasm (Fig. $4 A b, 1,025 \mathrm{sec}$ ). This was true for all cells expressing GFP-LA $\Delta 50$ /progerin, even after 
$\approx 2,100 \mathrm{sec}$ of observation from the metaphase/anaphase transition (SI Movie 4 and data not shown).

We also determined the extent of similarity of the cytoplasmic structures containing GFP-LA $\Delta 50$ /progerin found in HeLa cells in early $G_{1}$ with those seen in HGPS cells. In this regard, we found that endogenous LA, LB, and emerin colocalized with GFP-LA $\Delta 50 /$ progerin in similar cytoplasmic structures (Fig. 5 $A-I$ ). Upon treatment with FTI-277, GFP-LA $\Delta 50 /$ progerin was no longer detectable in the cytoplasm in late telophase/early $\mathrm{G}_{1}$ cells (Fig. 5J). Rather, it was distributed throughout the nucleoplasm, instead of being concentrated in the lamina region, as was the case for farnesylated GFP-LA $\Delta 50$ /progerin. The distribution of LB is also altered from its normal association with the surface of daughter cell nuclei to a nucleoplasmic pattern in FTI-277-treated cells (compare Fig. $5 N$ and $K$ ), colocalizing with GFP-LA $\Delta 50$ /progerin (Fig. $5 L$ ). This may be relevant, because plans for treating HGPS patients with FTIs are now under consideration. Cytoplasmic structures containing LA and LB were not detected in either GFP-LA $\Delta 50 /$ progerin-expressing cells treated with FTI-277 or GFP-LA-expressing cells in late telophase/early $\mathrm{G}_{1}$ (Fig. $5 \mathrm{~J}-\mathrm{O}$ and data not shown).

\section{Discussion}

The results of this study demonstrate that LA $\Delta 50 /$ progerin is stably farnesylated/carboxymethylated and that this leads to its abnormal association with membranes during mitosis. This defect delays the onset and progression of cytokinesis and the targeting of nuclear envelope components to daughter cell nuclei in late telophase/early $\mathrm{G}_{1}$. A delay in the progression through cytokinesis supports the finding by Cao et al. (see accompanying paper) that HeLa cells expressing GFP-LA $\Delta 50$ /progerin have an increased mitotic index, compared with those expressing GFPLA. These delays undoubtedly have a negative impact on the spatially and temporally regulated sequence of events that represent the hallmarks of normal cell cycle progression. In support of this hypothesis, we provide evidence that the $\mathrm{Rb}$ mediated progression into S-phase is impaired in HGPS cells.

One possible link between LA $\Delta 50$ /progerin expression and a decrease of $\mathrm{Rb}$ hyperphosphorylation may be INK4a, an inhibitor of cyclin D-dependent kinase activity (30). In support of this possibility, it has been shown that $\mathrm{LA} / \mathrm{C}$ is required for INK4amediated $\mathrm{G}_{1}$ arrest and that $\mathrm{LA} \Delta 50 /$ progerin can restore INK4a responsiveness in $L M N A^{-/-}$cells (31). Interestingly, INK4a is also thought to play a role in the aging process, possibly through reduction of the replicative potential of adult stem cells (30). This finding is of special interest, as recently it has been suggested that impaired adult stem cell function and tissue regeneration may be responsible for diseases caused by the numerous mutations in $L M N A$ (32).

In contrast to ZMPSTE $24^{-/-}$mice, in which decreased Rb levels have been described (33), $\mathrm{Rb}$ was still present in highly lobulated nuclei of HGPS cells. This is in agreement with the finding that $\mathrm{LA} \Delta 50$ /progerin does not decrease Rb stability as is the case for LA containing a mutation in the Zmpste24 cleavage site (31). Therefore, the HGPS phenotype may not only be attributable to the abnormal farnesylation of $\mathrm{LA} \Delta 50$ /progerin, but also to the 50 aa that have been deleted near its $\mathrm{C}$ terminus.

Our observations also extend previous studies that link normal and accelerated aging to mitotic defects, including impaired chromosome segregation and increases in bi- and multinucleated cells $(34,35)$. With respect to the latter, we have found that some mitotic cells expressing high levels of LA $\Delta 50$ /progerin show significant mitotic defects resulting in an even more delayed cytokinesis and in binucleated cells (SI Fig. 9 and SI Movie 5). This observation may help to explain the increased numbers of binucleated cells found in HGPS fibroblasts (see accompanying paper by Cao et al.; ref. 27).

Interestingly, it has been shown that low levels of LA $\Delta 50$ / progerin mRNA and protein are expressed in normal cells derived from very young and old people (36) and that binucleated cells in normal populations react with an LA $\Delta 50 /$ progerinspecific antibody (see accompanying paper by Cao et al.). These results, along with the data presented in this study, indicate that the defects in cytokinesis, nuclear assembly, and progression through the cell cycle attributable to the expression of the mutant LA responsible for HGPS, may also contribute to normal human aging.

\section{Materials and Methods}

Cell Culture, Lamin Expression, and Synchronization. Dermal fibroblasts from progeria patients (HGADFN003 and HGADFN127; The Progeria Research Foundation Cell Bank, Peabody, MA) and from normal controls (AG08470 and AG09309; Coriell Cell Repositories, Camden, NJ) were grown as described in ref. 5. HeLa Tet-on cell lines stably transfected with plasmids encoding either GFP-myc-LA or GFP-myc-LA $\Delta 50$ /progerin were maintained as described in ref. 19. Expression of GFP-fusion proteins was induced by the addition of $2 \mu \mathrm{g} / \mathrm{ml}$ doxycyclin to the growth medium for 24-50 h. For synchronizing HeLa Tet-on cells in mitosis, cells were incubated for $14 \mathrm{~h}$ in medium containing 0.2 $\mu \mathrm{g} / \mathrm{ml}$ nocodazole. Inhibition of protein farnesylation was achieved by incubating cells in medium containing $5 \mu \mathrm{M}$ FTI-277 (Calbiochem, San Diego, CA) for $50 \mathrm{~h}$.

Preparation of Mitotic Cell Extracts. Mitotically arrested HeLa Tet-on cells expressing either GFP-myc-LA or GFP-mycLA $\Delta 50 /$ progerin were harvested by mechanical shake-off, washed twice with PBS, incubated in $\approx 5$ vol of ice-cold hypotonic buffer (10 mM Hepes, $\mathrm{pH} 7.4 / 10 \mathrm{mM} \mathrm{NaCl} / 5 \mathrm{mM} \mathrm{MgCl} / 2$ $\mathrm{mM}$ EGTA/1 mM DTT/500 $\mu \mathrm{g} / \mathrm{ml}$ DNase $/ 200 \mu \mathrm{g} / \mathrm{ml}$ RNase) containing $1 \times$ Complete protease inhibitor mixture, EDTA-free (Roche, Basel, Switzerland), $20 \mu \mathrm{M}$ cytochalasin B (Sigma, St. Louis, MO), phosphatase inhibitors $[0.2 \mu \mathrm{M}$ calyculin A (Calbiochem) $/ 8 \mu \mathrm{M}$ microcystin $/ 0.5 \mathrm{mM} \beta$-glycerophosphate (both Sigma)] and homogenized in a glass-glass homogenizer. After the addition of $97 \mathrm{mM} \mathrm{NaCl}$, the samples were incubated in the absence or presence of $1.6 \%$ Triton X-100 for $15 \mathrm{~min}$ on ice and supernatant and pellet fractions were collected by centrifugation for $20 \mathrm{~min}$ at $19,000 \times g$.

Characterization of Farnesylation and Carboxymethylation. HeLa Tet-on cells expressing GFP-myc-LA $\Delta 50 /$ progerin were incubated for $36 \mathrm{~h}$ with $30 \mu \mathrm{M}$ AG, with or without FTI-277 $(3 \mu \mathrm{M})$, in culture medium. Cells were lysed in RIPA buffer (PBS/1\% Nonidet P-40/0.5\% sodium deoxycholate/0.1\% SDS/1 mM sodium orthovanadate $/ 0.1 \mathrm{mg} / \mathrm{ml} \mathrm{PMSF} / 1: 100$ protease inhibitor cocktail solution) (Santa Cruz Biotechnology, Santa Cruz, CA), and GFP-LA $\Delta 50 /$ progerin was immunoprecipitated with antiGFP [ab6556; Abcam (Cambridge, MA)] and analyzed by immunoblotting, using anti-AG (25) or anti-LA/C [clone 14; BD Biosciences (San Jose, CA)].

Carboxymethylation was determined essentially as described in ref. 12 . HeLa cells expressing GFP-myc-LA $\Delta 50$ /progerin were incubated with $50 \mu \mathrm{Ci} / \mathrm{ml}\left(\left[\right.\right.$ methyl $\left.\left.-{ }^{3} \mathrm{H}\right]\right)$ methionine for $16-20 \mathrm{~h}$ and then lysed in RIPA buffer (Santa Cruz Biotechnology). Proteins were immunoprecipitated consecutively from the cell lysate with anti-LB (C-20; Santa Cruz Biotechnology), antiLA/C (clone 14; BD Biosciences) or anti-GFP (AbCam, ab6556), separated by SDS/PAGE, and identified by immunoblotting with the appropriate antibodies. Protein bands were excised, incubated with $1 \mathrm{M} \mathrm{KOH}$ for $20 \mathrm{~h}$ in an Eppendorf (Boulder, CO) tube, and placed inside a scintillation vial containing scintillation mixture, and the diffusible counts were determined (basereleasable counts). The bands were then recovered and dissolved in perchloric acid- $\mathrm{H}_{2} \mathrm{O}_{2}$, and nonreleasable counts were determined by liquid scintillation. Relative methylation was calculated by using the following formula: Carboxymethylation $\%=(($ Base 
Releasable counts - Blank/Non-Base Releasable counts Blank) $\times($ no. methionines -1$)) \times 100$.

Immunofluorescence. Cells grown on cover slips were fixed either for $5 \mathrm{~min}$ at $-20^{\circ} \mathrm{C}$ in methanol or for $15 \mathrm{~min}$ at room temperature in either 3\% paraformaldehyde in PBS or $3.7 \%$ formaldehyde in PBS, followed by extraction in $0.1 \%$ Triton $\mathrm{X}-100$ in PBS for $5 \mathrm{~min}$ at room temperature, and processed for immunofluorescence as described in ref. 19. Rabbit antibodies used were directed against LA [1:1,000; generated against a synthetic peptide with the sequence VTVTRSYRSVGGSG, which is not present in LA $\Delta 50$ /progerin; PRB-113C (Covance, Princeton, NJ)], LB (1:200; ref. 9), phosphoRb(Ser-807/811) (1:100; Cell Signaling Technology, Danvers, MA) and cyclin D1 [1:200; H-295 (Santa Cruz Biotechnology)]; mouse monoclonal antibodies were directed against emerin [1:50; NCL-emerin (Novocastra, Newcastle, U.K.)] and Rb [1:100; 4H1 (Cell Signaling)]; rat anti-LA/C (1:1,200; no. 320, generated against bacterial expressed full-length human LA), and goat-anti LA/C [1:200; N-18 (Santa Cruz Biotechnology)]. The secondary antibodies used were anti-rabbit IgG-Alexa Fluor 488 and 568, anti-rat IgG-Alexa Fluor 488 or 568, anti-mouse IgG-Alexa Fluor 488 and 568, and anti-goat IgG-Alexa Fluor 568 (all 1:400; Molecular Probes). DNA was stained with Hoechst dye, and

1. Hutchinson J (1886) Medicochir Trans 69:473-477.

2. Gilford M (1904) Practitioner 73:188-217.

3. De-Sandre-Giovannoli A, Bernard R, Cau P, Navarro C, Amiel J, Boccaccio I, Lyonnet S, Stewart CL, Munnich A, Le-Merrer M, Levy N (2003) Science 300:2055.

4. Eriksson M, Brown WT, Gordon LB, Glynn MW, Singer J, Scott L, Erdos MR, Robbins CM, Moses TY, Berglund P, et al. (2003) Nature 423:293-298.

5. Goldman RD, Shumaker DK, Erdos MR, Eriksson M, Goldman AE, Gordon LB, Gruenbaum Y, Khuon S, Mendez M, Varga R, Collins FS (2004) Proc Natl Acad Sci USA 101:8963-8968.

6. Mounkes LC, Stewart CL (2004) Curr Opin Cell Biol 16:322-327.

7. Ackerman J, Gilbert-Barness E (2002) Pediatr Pathol Mol Med 21:1-13.

8. Broers JL, Ramaekers FC, Bonne G, Yaou RB, Hutchison CJ (2006) Physiol Rev 86:967-1008.

9. Moir RD, Montag-Lowy M, Goldman RD (1994) J Cell Biol 125:1201-1212.

10. Goldman RD, Gruenbaum Y, Moir RD, Shumaker DK, Spann TP (2002) Genes Dev 16:533-547.

11. Kitten GT, Nigg EA (1991) J Cell Biol 113:13-23.

12. Sinensky M, Fantle K, Trujillo M, McLain T, Kupfer A, Dalton M (1994) J Cell Sci 107:61-67.

13. Bergo MO, Gavino B, Ross J, Schmidt WK, Hong C, Kendall LV, Mohr A, Meta M, Genant H, Jiang Y, et al. (2002) Proc Natl Acad Sci USA 99:1304913054.

14. Corrigan DP, Kuszczak D, Rusinol AE, Thewke DP, Hrycyna CA, Michaelis S, Sinensky MS (2005) Biochem J 387:129-138.

15. Pendas AM, Zhou Z, Cadinanos J, Freije JM, Wang J, Hultenby K, Astudillo A, Wernerson A, Rodriguez F, Tryggvason K, Lopez-Otin C (2002) Nat Genet 31:94-99.

16. Rusinol AE, Sinensky MS (2006) J Cell Sci 119:3265-3272.

17. Liu B, Wang J, Chan KM, Tjia WM, Deng W, Guan X, Huang JD, Li KM, Chau PY, Chen DJ, et al. (2005) Nat Med 11:780-785.

18. Manju K, Muralikrishna B, Parnaik VK (2006) J Cell Sci 119:2704-2714. confocal images were taken with an LSM 510 META (Zeiss, Thornwood, NY).

Immunoblotting. Cell fractions were denatured in Laemmli buffer, separated by SDS/PAGE, and immunoblotted as described in ref. 5. Primary antibodies used were rat anti-LA/C $(1: 1,000$; no. 270 ; ref. 5$)$, rabbit anti-LB (1:1,200; ref. 9), rabbit anti-EGFP [1:5,000; ab6556 (AbCam)], mouse anti-AG (1:5,000; ref. 25), mouse anti-LA/C [1:5,000; clone 14 (BD Biosciences)], mouse anti-emerin [1:300; NCL-emerin (Novocastra)], and goat anti-LB [1:1,000; C-20 (Santa Cruz Biotechnology)]. Secondary antibodies were goat anti-rat IgG, anti-rabbit IgG, and antimouse $\mathrm{IgG}$ conjugated to horseradish peroxidase (all 1:1,000; Kirkegaard and Perry Laboratories, Gaithersburg, MD). Detection was achieved by using 4-chloro-1-naphtol/ $\mathrm{H}_{2} \mathrm{O}_{2}$.

Statistical Analysis. A two-tailed homoscedastic Student's $t$ test was used to compare mean levels. $P<0.01$ was considered statistically significant.

This work was supported by the Kentucky Lung Cancer Research Program (D.A.A. and H.P.S.), the U.S. National Institute for General Medical Sciences (H.P.S.), a Schroedinger Fellowship from the Austrian Research Foundation (to T.D.), the U.S. National Cancer Institute, the U.S. National Institute on Aging, and the Ellison Foundation.

19. Shumaker DK, Dechat T, Kohlmaier A, Adam SA, Bozovsky MR, Erdos MR, Eriksson M, Goldman AE, Khuon S, Collins FS, et al. (2006) Proc Natl Acad Sci USA 103:8703-8708.

20. Clements L, Manilal S, Love DR, Morris GE (2000) Biochem Biophys Res Commun 267:709-714.

21. Ozaki T, Saijo M, Murakami K, Enomoto H, Taya Y, Sakiyama S (1994) Oncogene 9:2649-2653.

22. Giacinti C, Giordano A (2006) Oncogene 25:5220-5227.

23. Zarkowska T, Mittnacht S (1997) J Biol Chem 272:12738-12746.

24. Parish CA, Rando RR (1996) Biochemistry 35:8473-8477.

25. Troutman JM, Roberts MJ, Andres DA, Spielmann HP (2005) Bioconjug Chem 16:1209-1217

26. Dechat T, Vlcek S, Foisner R (2000) J Struct Biol 129:335-345.

27. Cao K, Capell BC, Erdos MR, Djabali K, Collins FS (2007) Proc Natl Acad Sci USA 104:4949-4954.

28. Tsai MY, Wang S, Heidinger JM, Shumaker DK, Adam SA, Goldman RD, Zheng Y (2006) Science 311:1887-1893.

29. Dechat T, Gajewski A, Korbei B, Gerlich D, Daigle N, Haraguchi T, Furukawa K, Ellenberg J, Foisner R (2004) J Cell Sci 117:6117-6128.

30. Kim WY, Sharpless NE (2006) Cell 127:265-275.

31. Nitta RT, Jameson SA, Kudlow BA, Conlan LA, Kennedy BK (2006) Mol Cell Biol 26:5360-5372.

32. Gotzmann J, Foisner R (2006) Histochem Cell Biol 125:33-41.

33. Varela I, Cadinanos J, Pendas AM, Gutierrez-Fernandez A, Folgueras AR, Sanchez LM, Zhou Z, Rodriguez FJ, Stewart CL, Vega JA, et al. (2005) Nature 437:564-568.

34. Baker DJ, Jeganathan KB, Cameron JD, Thompson M, Juneja S, Kopecka A, Kumar R, Jenkins RB, de Groen PC, Roche P, van Deursen JM (2004) Nat Genet 36:744-749.

35. Ly DH, Lockhart DJ, Lerner RA, Schultz PG (2000) Science 287:2486-2492.

36. Scaffidi P, Misteli T (2006) Science 312:1059-1063. 